\title{
Fully coupled dynamic analysis of a FPSO and its MWA system with
}

\author{
mooring lines and risers
}

\author{
ChunyanJi,Yong Cheng*, Qi Yan, GeogreWang \\ School of naval architecture and ocean engineering, Jiangsu University of Science and Technology, \\ Zhenjiang, 212003, China
}

\begin{abstract}
The middle water arch (MWA) is an innovation that is developed for floating production storage and offloading (FPSO). During simulating structural global motion in time domain, the fully coupled hull, mooring lines, tendons and risers technique is widely recognized as a unique method to understand the accurate hydrodynamic interactions and coupling effects. This paper investigates the dynamic response characteristics of a single point turret-moored FPSO-MWA system under the combined parallel wave, wind and current conditions by using coupled time domain analysis code AQWA. The complex nonlinear issues e.g. forces nonlinearity, mooring nonlinearity, motion nonlinearity are included in the numerical model. To illustrate the hydrodynamic interactions and coupling effects of FPSO, MWA and mooring system, the numerical solutions are systematically compared with those from the single body mooring system without considering the effects of MWA mooring system. The results indicate that the MWA system has not only an important influence on the top tensions and shapes of risers, but also have some contribution to motion responses of the FPSO and mooring lines. Furthermore, the results reveal that the favorable motion performance of MWA in the multi-body system may provide some economic benefits due to the possible decrease of fabrication cost and work-off days.
\end{abstract}

Keywords: Floating production storage and offloading (FPSO), middle water arch (MWA), multi-body hydrodynamic interaction, nonlinear, time domain

\section{Introduction}

Floating production storage and offloading (FPSO), spar, tension leg platform (TLP) and semi-submersible (SEMI) are popular production platforms. These platforms or vessels must be anchored through a mooring system. For FPSO, single point turret-moored [1] is often used and submerged turret production (STP) is the first choice in South China Sea. A STP mooring system which consists of mooring lines, risers and a middle water arch (MWA), has the advantages of smaller top tension, less construction difficulty and favorable motion performance. Prediction of motion responses of FPSO-STP is challenging because of the interaction of wave, wind and current with hulls, flow lines, tension legs and flexible risers.

The dynamic responses of FPSOs under wind, wave and current actions have been studied by some researchers. Based on the conventional uncoupled theory, Wichers and Devlin [2] derived the motion equations of FPSO and its mooring lines in time domain. Lee [3] also adopted the simple uncoupled analysis for investigating the hydrodynamic performance of tandem moored tankers. In the uncoupled analysis, the mooring and risers are treated as massless linear or nonlinear spring, and the hull motions as well as the top tension of mooring lines are solved from static mooring load [4]. However, as the water depth increases, the uncoupled analysis becomes conservative. The coupled dynamic analysis is considered a better alternative as it takes into 
account the fully hydrodynamic interactions among hulls, mooring lines, tendons and risers. Zhang Lei et al. [5] studied the low frequency drift forces, horizontal motions and mooring system design of a multi-point moored FPSO in bi-directional swell and wind-sea conditions by using Newman's approximation. They also conducted relevant model experiments and validate the numerical solutions. Tahar and Kim [6] developed numerical solutions for the coupled-dynamic analysis of a deepwater Spar platform with polyester mooring lines, and then these numerical results were compared with those with equivalent elastic lines. Yang et al. [7] further improved the full time-domain coupled analysis program to include the nonlinear effects, avoiding the excessive artificial participation. Some cases with floating hemisphere, mooring/riser models and mooring truss Spar indicated that their method gives reliable results for both low frequency and wave frequency motion.

In order to accurately evaluate motion responses of a FPSO with its MWA, multi-body hydrodynamic interaction should be studied. One way to tackle this problem is to use a frequency-domain method given by Korloo et al. [8], Fang and Kim [9], and Huijsmans [10]when determining the response amplitude operator (RAO) of the floating body and pertinent response parameters in a steady state condition. However, frequency domain method can be cumbersome to implement and computationally inefficient for solving the coupled effects of moorings and platforms as well as the interaction between two floating bodies. Furthermore, the frequency domain model ignore the complex nonlinear issues e.g. forces nonlinearity, mooring nonlinearity, motion nonlinearity. Thus, many researchers have devoted significant efforts to the time domain coupled analysis of multi-body hydrodynamic interaction between these platforms. Buchner et a1. [11]developed a time-domain model to execute a coupled dynamic analysis of LNG-FPSO with alongside moored LNGC in close proximity, showing that a free surface lid in the multiple-body diffraction analysis can significantly improve the prediction of drift force and relative sway/yaw motions. Koo and Kim [12] introduced the exact and approximation methods to evaluate the contribution of off-diagonal hydrodynamic interaction terms on the relative motions of two vessels. The most remarkable conclusion is that there may be large discrepancy for predicting the actual relative motion between the two methods due to the role of cross-coupling terms of coefficient matrix. Zhao et al. [13-15] investigated the time-domain hydrodynamic interaction between the FLNG vessel and the LNG carrier in side by side and tandem offloading configuration in which the mechanical coupling effects between the floating bodies and their connection systems are included in the coupled analysis model. They also conducted systematic experiments by using small-scale models to validate their numerical solutions.

Although the multi-body coupled analysis was much studied, studies accumulated are still far from enough for the motions of a FPSO with its MWA system under wind, wave and current actions. In this paper, a coupled analysis model of the multi-body problem is established in real sea states, taking into consideration the fully coupled effects in the time domain including viscous damping contributed by many slender members such as mooring lines, tendons and risers as well as hydrodynamic interactions between FPSO and MWA. To illustrate these coupled effects, the numerical solutions without considering multi-body interactions also are established and executed for comparison. Finally, the main conclusions of this paper are drawn.

\section{Description of the FPSO with its MWA system}

A typical FPSO consists of oil storage vessel, mooring system, riser system and production 
equipment. Fig. 1 and Table 1 show the structural components and summarize the mass and dimension information for FPSO. The design and construction of the suction anchor depends on the hull's ability to maintain a stationary position. Currently, the FPSO operates in waters over $1000 \mathrm{~m}$ deep, and plans are underway to move to the depths of $2200 \mathrm{~m}$ in South China Sea. Thus, designing low-risk and efficient position-keeping mooring systems has widely intrigued researches and engineers.

The mooring system of submerged turret production (STP) includes 12 mooring lines, riser system and middle water arch (MWA), which is expected to provide buoyancy to keep desired curvature and shape of these flexible risers. All mooring lines are separated into three groups and symmetrically arranged on the turret, which as shown in Fig. 2. Each group is $120^{\circ}$ from another and the lines of each group are in $5^{0}$ spread azimuth. Each line consists of an upper chain section, a middle cable section and a bottom chain section. The initial tension of each line is $1.2 \times 10^{6} \mathrm{~N}$, and the mooring line properties are listed in Table 2.

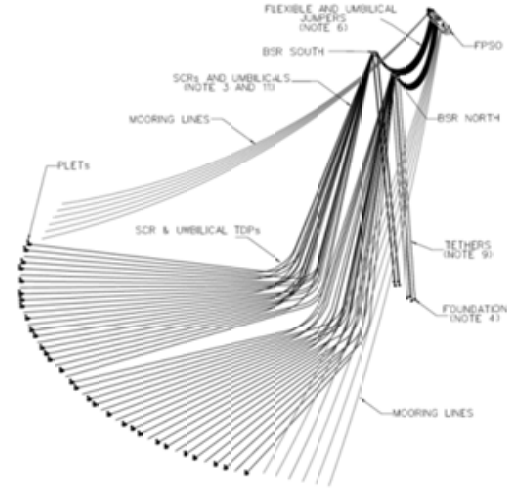

Fig. 1 FPSO production system

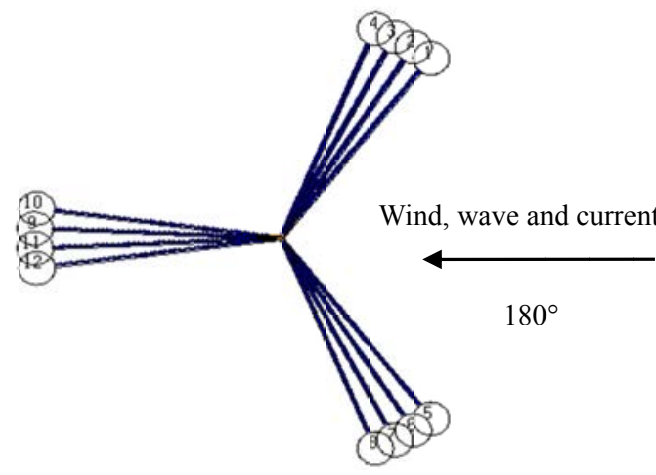

Fig. 2 Mooring configuration and environmental condition

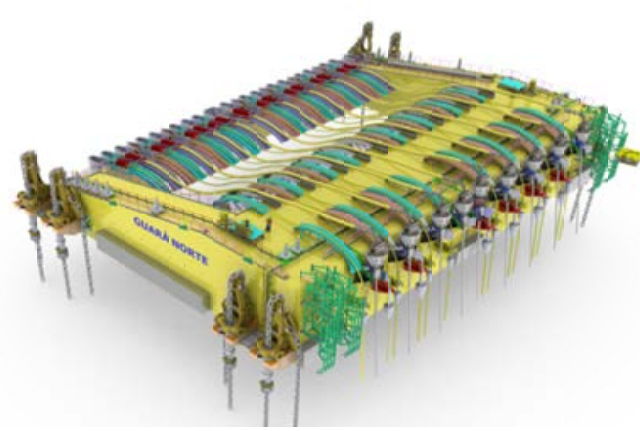

Fig. 3 The layout of MWA

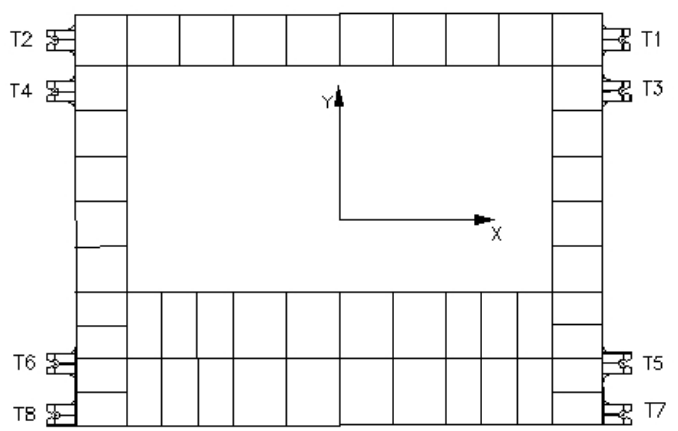

Fig. 4 Tendons configuration of MWA

In order to control the motion responses of flexible risers and power cables, the MWA is installed at desired submergence depth by tension legs moored to seabed, as shown in Fig. 3. The higher side of a MWA with single slope is attached to the manifold system and the lower side is connected to the hull. Some flexible risers are divided two groups, where one group is employed to connect the FPSO to MWA, whereas another group is used to connect MWA to seabed, and each group is composed of twenty-two risers for the exploration and production of the oil and natural gas. The relevant properties of the risers are shown in Table 3, and these risers are protected by the MWA from action of wave and current. The intial tension of each riser is $1.2 \times$ $10^{6} \mathrm{~N}$. In this paper, the MWA is placed at the submergence depth of $250 \mathrm{~m}$, and the main 
characteristic parameters are listed in Table 4. The employment of the MWA potentially increases of the mooring restoring stiffness because the several thousand tons tension can be resisted by tension legs. The MWA employ 8 tension legs, which are shown in Fig. 4. The coordinates of the top porch for all tendons are summarized in Table 5, and the properties of each tension leg are illustrated in Table 6. In addition, only upper mooring lines need to be retracted when storms come and the gravity of ground cables is resisted by buoys. Therefore, investigating the coupled dynamic responses between the FPSO and MWA has become more challenging.

Tab. 1 The main parameters of a FPSO

\begin{tabular}{ccccc}
\hline Dimension & Symbol & Unit & Ballast condition & Full-laden condition \\
\hline Length & LOA & $\mathrm{m}$ & 329 & 329 \\
Waterline length & LWL & $\mathrm{m}$ & 309.177 & 325 \\
Breadth & $\mathrm{B}$ & $\mathrm{m}$ & 64.8 & 64.8 \\
Height & $\mathrm{D}$ & $\mathrm{m}$ & 32 & 32 \\
Displacement & $\Delta$ & $\mathrm{t}$ & 187328 & 389688 \\
Draft & $\mathrm{T}$ & $\mathrm{m}$ & 9.858 & 19.891 \\
Centre of gravity above base & $\mathrm{VCG}$ & $\mathrm{m}$ & 16.272 & 17.807 \\
X-coordinate of centre of gravity & LCG & $\mathrm{m}$ & 167.943 & 163.738 \\
Buoyant centre above base & VCB & $\mathrm{m}$ & 5.004 & 10.159 \\
X-coordinate of centre of buoyant & $\mathrm{LCB}$ & $\mathrm{m}$ & 167.943 & 163.738 \\
\hline centre & & & &
\end{tabular}

Tab. 2 Mooring lines properties

\begin{tabular}{llllllll}
\hline Item & Length & Diameter & $\begin{array}{l}\text { Weight } \\
\text { in water } \\
\mathrm{kg} / \mathrm{m}\end{array}$ & $\begin{array}{l}\text { Breaking } \\
\text { strength } \\
\mathrm{N}\end{array}$ & $\begin{array}{l}\text { Axial } \\
\text { stiffness } \\
\mathrm{mm}\end{array}$ & $\begin{array}{l}\text { Added mass } \\
\text { coefficient }\end{array}$ & $\begin{array}{l}\text { Drag } \\
\text { coefficient }\end{array}$ \\
\hline R4S chain (upper) & 160 & 120 & 263.4 & $1.58 \mathrm{E} 7$ & $2.13 \mathrm{E} 9$ & 2.2 & 2.0 \\
Polyester (middle) & 3300 & 232 & 9.7 & $1.57 \mathrm{E} 7$ & $4.29 \mathrm{E} 8$ & 1.8 & 1.0 \\
R4S Chain (bottom) & 220 & 120 & 263.4 & $1.58 \mathrm{E} 7$ & $2.13 \mathrm{E} 9$ & 2.2 & 2.0 \\
\hline
\end{tabular}

Tab. 3 Riser properties

\begin{tabular}{ccccccccc}
\hline Item & $\begin{array}{c}\text { Length } \\
(\mathrm{m})\end{array}$ & $\begin{array}{c}\text { Outer } \\
\text { diameter } \\
(\mathrm{mm})\end{array}$ & $\begin{array}{c}\text { Inner } \\
\text { diameter } \\
(\mathrm{mm})\end{array}$ & $\begin{array}{c}\text { Axial } \\
\text { stiffness }\end{array}$ & $\begin{array}{c}\text { Bending } \\
\text { stiffness }(\mathrm{N})\end{array}$ & $\begin{array}{c}\text { Weight } \\
\text { in water }\end{array}$ & $\begin{array}{c}\text { Added } \\
\text { mass }\end{array}$ & $\begin{array}{c}\text { Drag } \\
\text { coefficient }\end{array}$ \\
\hline Flexible 1 & 721 & 220 & 153 & $1.256 \mathrm{E} 8$ & $2.313 \mathrm{E} 4$ & 21.1 & 1.1 & 1.0 \\
Flexible 2 & 2837 & 220 & 153 & $1.256 \mathrm{E} 8$ & $2.313 \mathrm{E} 4$ & 21.1 & 1.1 & 1.0 \\
\hline
\end{tabular}

Tab. 4 The main parameters of the MWA

\begin{tabular}{ccc}
\hline Designation & Unit & Values \\
\hline Length & $\mathrm{m}$ & 52
\end{tabular}




\begin{tabular}{ccc} 
Width & $\mathrm{m}$ & 40 \\
Displacement & $\mathrm{t}$ & 9566 \\
Weight & $\mathrm{t}$ & 7500 \\
X-coordinate of centre of gravity & $\mathrm{m}$ & -7.55 \\
z-coordinate of centre of gravity & $\mathrm{m}$ & 4.0 \\
X-coordinate of buoyant centre & $\mathrm{m}$ & -7.54 \\
z-coordinate of centre of buoyant centre & $\mathrm{m}$ & 4.5 \\
\hline
\end{tabular}

Tab. 5 The coordinate of Porch

\begin{tabular}{cccc}
\hline Number & $\mathrm{X}(\mathrm{m})$ & $\mathrm{Y}(\mathrm{m})$ & $\mathrm{Z}(\mathrm{m})$ \\
\hline 1 & 26.000 & 17.500 & -260.00 \\
2 & -26.000 & 17.500 & -260.00 \\
3 & 26.000 & 12.500 & -260.00 \\
4 & -26.000 & 12.500 & -260.00 \\
5 & 26.000 & -14.00 & -260.00 \\
6 & -26.000 & -14.00 & -260.00 \\
7 & 26.000 & -19.00 & -260.00 \\
8 & -26.000 & -19.00 & -260.00 \\
\hline
\end{tabular}

Tab. 6 The parameters of tendons

\begin{tabular}{ccc}
\hline Item & Values & Unit \\
\hline Pre-tension & 2066 & $\mathrm{mt}$ \\
Number of tendons & 8 & $\mathrm{n}$ \\
Length & 1950 & $\mathrm{~m}$ \\
Diameter & 1.067 & $\mathrm{~m}$ \\
Wall thickness & 40 & $\mathrm{~mm}$ \\
Elastic modulus & $2.07 \mathrm{E}+11$ & $\mathrm{pa}$ \\
Axial stiffness & $2.71 \mathrm{E}+10$ & $\mathrm{~N}$ \\
Limit yield stress & 552 & $\mathrm{Mpa}$ \\
\hline
\end{tabular}

\section{Mathematical formulation}

\subsection{Hull hydrodynamics}

The FPSO and its MWA system are modeled as panel elements shown in Fig. 5, and subsequently the potential theory is applied to the water waves in the numerical model. The first order velocity potential can be expressed by the sum of the incident potential $\Phi_{I}(x, y, z, t)$, the diffraction $\Phi_{D}(x, y, z, t)$ and the radiation potential $\Phi_{R}(x, y, z, t)$ and the radiation potential $\Phi_{R}(x, y, z, t)$ can be further decomposed as follows:

$\Phi(x, y, z, t)=\operatorname{Re}\left\{\left(\phi_{I}(x, y, z)+\phi_{D}(x, y, z)-i \omega \sum_{j=1}^{N} \zeta_{j} \phi_{j}\right) e^{-i \omega t}\right\}$ 
where Re represents the real part of the argument. $i^{2}=-1 . t$ and $\omega$ are the time and the wave frequency, respectively. $\phi_{j}$ is the radiation potential corresponding to the unit-amplitude $j$-th motion, and $\zeta_{j}$ is the amplitude of $j$-th motion. Once each part of the potential in Eq. (1) is solved with the help of Green's second identity, we can obtain the wave hydrodynamic information involving the first order wave exciting force $F_{j}^{1}$, added mass $a_{i j}$ and radiation damping $b_{i j}$ as follows

$$
\begin{aligned}
& F_{j}^{1}=\operatorname{Re}\left[\iint_{S_{B}} i \omega \rho\left(\phi_{I}+\phi_{D}\right) n_{j} d s\right] \\
& a_{i j}(\omega)=\operatorname{Re}\left[\iint_{S_{B}} \rho \phi_{i} n_{j} d s\right] \\
& b_{i j}(\omega)=\operatorname{Re}\left[\iint_{S_{B}} \rho \omega \phi_{i} n_{j} d s\right]
\end{aligned}
$$

where $\rho$ and $n_{j}$ denote the water density, generalized surface normal vector for $j$-th direction, respectively.
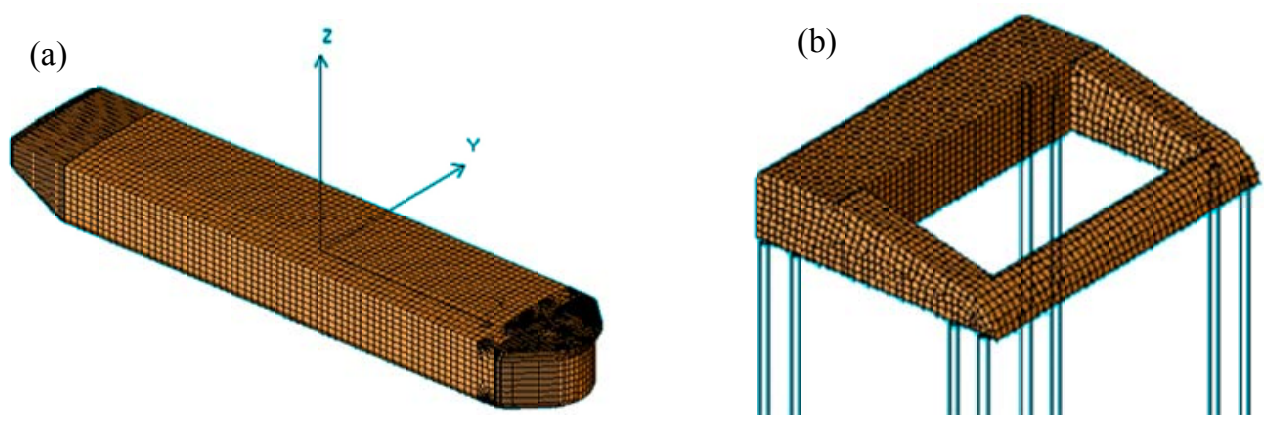

Fig. 5 (a) FPSO panel model, (b) MWA panel model

Besides the diffraction/radiation theory is applied to calculate the hydrodynamic forces of hull surfaces, the slender members such as mooring lines, tendons and risers is simulated by Morison equation as

$$
F_{M f}=C_{I} \rho \frac{\pi D^{2} l}{4} \dot{u}-C_{a} \rho \frac{\pi D^{2} l}{4} \ddot{x}+\frac{1}{2} \rho C_{d}(u-\dot{x})|(u-\dot{x})| D l
$$

where $D, l, C_{a}, C_{I}$ and $C_{d}$ are the diameter, length, added mass coefficient, initial force coefficient and drag coefficient of Morison element, respectively. $u / \dot{u}$ and $\dot{x} / \ddot{x}$ represent the water wave velocity/acceleration and structural velocity/acceleration, respectively. The viscous damping induced by the two platforms can significantly reduce the motion responses of multiple bodies near the natural frequencies, and thus the viscous damping coefficient $C_{p}$ can be estimated by using the Morison drag item as:

$$
C_{p}=\frac{1}{2} C_{d}^{p} \rho A_{p}
$$


where $C_{d}^{p}$ and $A_{p}$ are drag coefficient of platforms and projected area with ignoring the sheltering effects.

\subsection{Governing equation of the mooring lines/risers/tension legs}

With the increasing of water depth, the impact of damping induced by moorings/risers/tension legs on the motions of platforms is increasingly apparent. Furthermore, the responses of the moorings/risers/tension legs are also affected in order to restrict the drift motions of the platforms. Therefore, dynamic analysis of mooring lines/risers/tendons is necessary for the above-mentioned coupling motion. In this paper, it is assumed that there is no torque and applied external moment on a mooring line/riser/tendon, and the mooring line/riser/tension leg is modeled as some beam elements based on the slenderrod theory. Thus the motion equation of each element is derived as follows:

$$
\left(\boldsymbol{M}_{m}+\boldsymbol{M}_{m a}\right) \ddot{\boldsymbol{X}}_{m}+\left(\boldsymbol{C}_{m}+\boldsymbol{C}_{m a}\right) \dot{\boldsymbol{X}}_{m}+\boldsymbol{R}_{m} \boldsymbol{X}_{m}=\boldsymbol{F}_{g}+\boldsymbol{F}_{s}+\boldsymbol{F}_{d}
$$

where $\boldsymbol{M}_{m}, \boldsymbol{M}_{m a}, \boldsymbol{C}_{m}, \boldsymbol{C}_{m a}$ and $\boldsymbol{R}_{\boldsymbol{m}}$ represent the element mass matrix, added mass matrix, damping matrix,added damping matrix and stiffness matrix, respectively. $\boldsymbol{X}_{\boldsymbol{m}}$ is the element displacement vector. $\boldsymbol{F}_{g}, \boldsymbol{F}_{s}$ and $\boldsymbol{F}_{d}$ denote the element weight,hydrostatic forces including buoyancy forces, external and internal pressures, hydrodynamic force.It is noted that each node of mooring line elements has three degrees including one inline and two normal translation freedoms, while all the rotation and translation motions are considered except these about the axial direction for tendon elements, which means each node has four degrees including surge, sway, roll and pitch. In this case, each mooring lines and tendons is divided into 60 and 14 elements which ensure adequate calculation precision through tests.

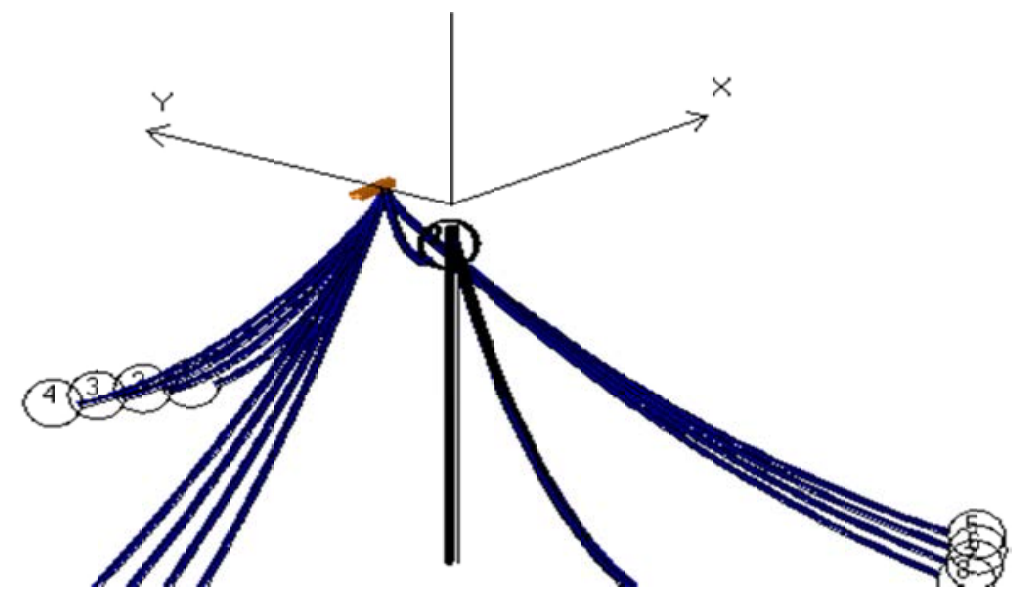

Fig. 6 Coupled analysis model

\subsection{Rigid body motion}

The model for the coupled analysis of the FPSO with its MWA system is shown in Fig.6. This paper only establishes the fully coupled model of a MWA system to investigate the hydrodynamic interaction between the FPSO and MWA. The low frequency motion responses obtained from the frequency domain method is usually validated to be a non-conservative estimation because of ignoring the resonant response and some nonlinear factors i.e. damping contribution of mooring lines/tendons, viscous damping and initial force effects. Thus, the time domain motion equations of multiple bodies are adopted for considering the coupling effects of 
the structures, moorings, risers and tendons. When the FPSO with its MWA system is simultaneously subjected to the action of wave, wind and current, the motion equations in time domain coupled analysis are formulated as follows:

$$
[M+a(\infty)]\{\ddot{\xi}\}+[B]\{\dot{\xi}\}+[K]\{\xi\}+\int_{0}^{t} h(t-\tau)\{\dot{\xi}\} d \tau=F_{w a}+F_{c u}+F_{w i}+F_{m o}
$$

where $M, a(\infty), B$, and $K$ are generalizedmass matrix, added mass matrix, damping matrix, and hydrostatic restoring stiffness matrix, respectively. $F_{w a}, F_{c u}, F_{w i}$ and $F_{m o}$ represent the wave force, wind force, current force and mooring force, respectively. $h(\tau)$ is the retardation function computed by transform of the frequency-dependent added-mass $a(\omega)$ and damping $b(\omega)$ :

$h(\tau)=\frac{1}{2 \pi} \int_{-\infty}^{\infty}[b(\omega)+i \omega a(\omega)] e^{i \omega t} d \omega$

here $\omega$ is wave frequency.

\section{Numerical results}

\subsection{Motions of FPSO for fully coupled model}

In order to calculate the motion response of the FPSO and its MWA system and investigate the coupled effects of environment conditions, FPSO, MWA and mooring system, the three hours numerical simulation is conducted without and with considering the hydrodynamic interactions between the FPSO and the MWA. During the time domain simulation, a convolution method, rigorously transforming the frequency-dependent hydrodynamic coefficients to time domain, is adopted. The collinear wave-wind-current environmental conditions are studied here with the heading of $180^{\circ}$. The typical working condition is described i.e. a three-parameter JONSWAP spectrum with a significant wave height of $3.1 \mathrm{~m}$, a spectrum peak of $9.65 \mathrm{~s}$ and a peak enhancement factor of 3.3. The time-varying wind forces are generated based on the API wind spectrum with a mean speed of $12 \mathrm{~m} / \mathrm{s}$ at $10 \mathrm{~m}$ height. The current velocity near the free surface is $0.4 \mathrm{~m} / \mathrm{s}$. The wind and current coefficients for FPSO in $x$ direction are $-7.79 \times 10^{2} \mathrm{~N} \cdot \mathrm{s}^{2} / \mathrm{m}^{2}$ and $-5.63 \times 10^{4} \mathrm{~N} \cdot \mathrm{s}^{2} / \mathrm{m}^{2}$, respectively. The time domain results for surge, sway, heave, roll, pitch and yaw responses of the FPSO based on the fully FPSO, MWA, mooring, riser and tendon coupled model are plotted in Fig. 7(a)-(f). In order to confirm the feasibility of the present method, the motion responses obtained from the coupled analysis code SIMO [16] are also included in these figures as a comparison with the present results. As can be seen from these figures, the results given by the present method and SIMO agree well with each other. In addition, the FPSO has an obvious surge slow drift displacement about $-95 \mathrm{~m}$ owing to the hydrodynamic interactions between the FPSO and MWA and the mechanical coupling effects between hulls, mooring system and riser system. After that point, the surge responses are controlled by reciprocating vibration. The sway always performs a slow drift motion, while the heave, roll and pitch responses are dominated by wave frequency motion. The yaw responses reaches its maximum around $\mathrm{t}=1000 \mathrm{~s}$ and then decreases monotonically with the increase of time $t=1000 \mathrm{~s}$. Accordingly, at $\mathrm{t}=1000 \mathrm{~s}$, the yaw responses are the largest. On the other hand, the effects of the MWA system on the motion of the FPSO andthe top tension of the mooring lines and flexible risers are discussed in detailas the following sections. 

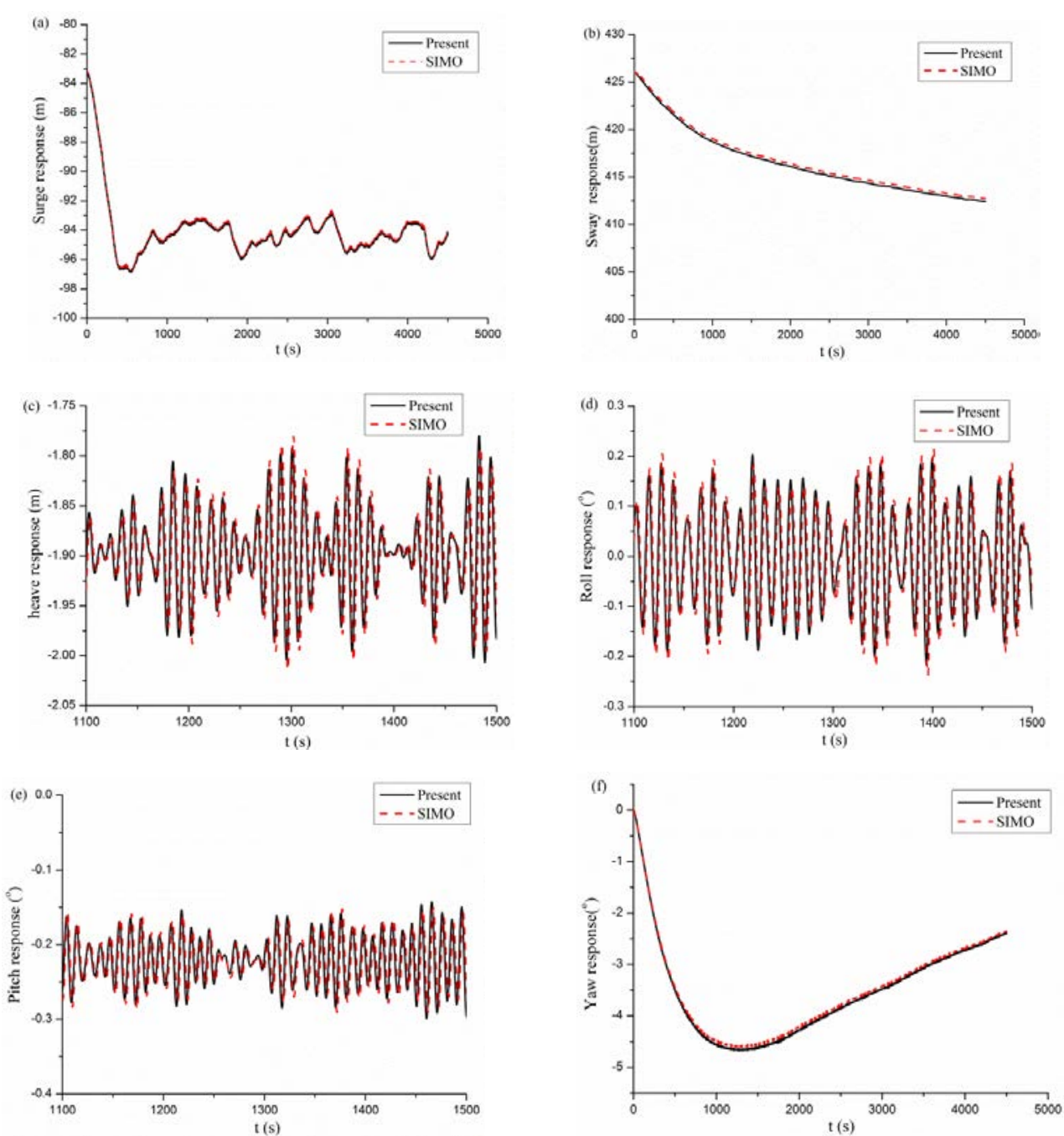

Fig. 7 The time series of the FPSO motion using fully coupled model. (a) surge (b) sway (c) heave (d) roll (c) pitch

(d) yaw

Next, the response amplitude operator (RAO) comparisons between the present method and numerical tool SIMO [16] are given in Fig. 8a-f for three different incident wave angles $\theta=0^{\circ}, 30^{\circ}$ and $60^{\circ}$. In these figures, the symbols denote the results calculated using the present method, while the lines stand for the solutions of code SIMO. The agreement between the two independent numerical tools is good. Fig.8a-f shows the surge, sway, heave, roll, pitch and yaw diffraction forces of the FPSO in frequency domain as a function of wave frequency. It can be seen from these figures that the total surge diffraction force decreases monotonically with increasing incident wave angle, the opposite is true for the total sway and yaw diffraction force. As the incident wave angle increases, the peak values of heave and pitch diffraction forces increase, while the roll is reduced. The wave frequency for the maximum diffraction forces vary with the incident wave angles, but the trends of the diffraction force versus the wave frequency for the incident wave angles $0^{\circ}, 30^{\circ}$, and $60^{\circ}$ are similar. 

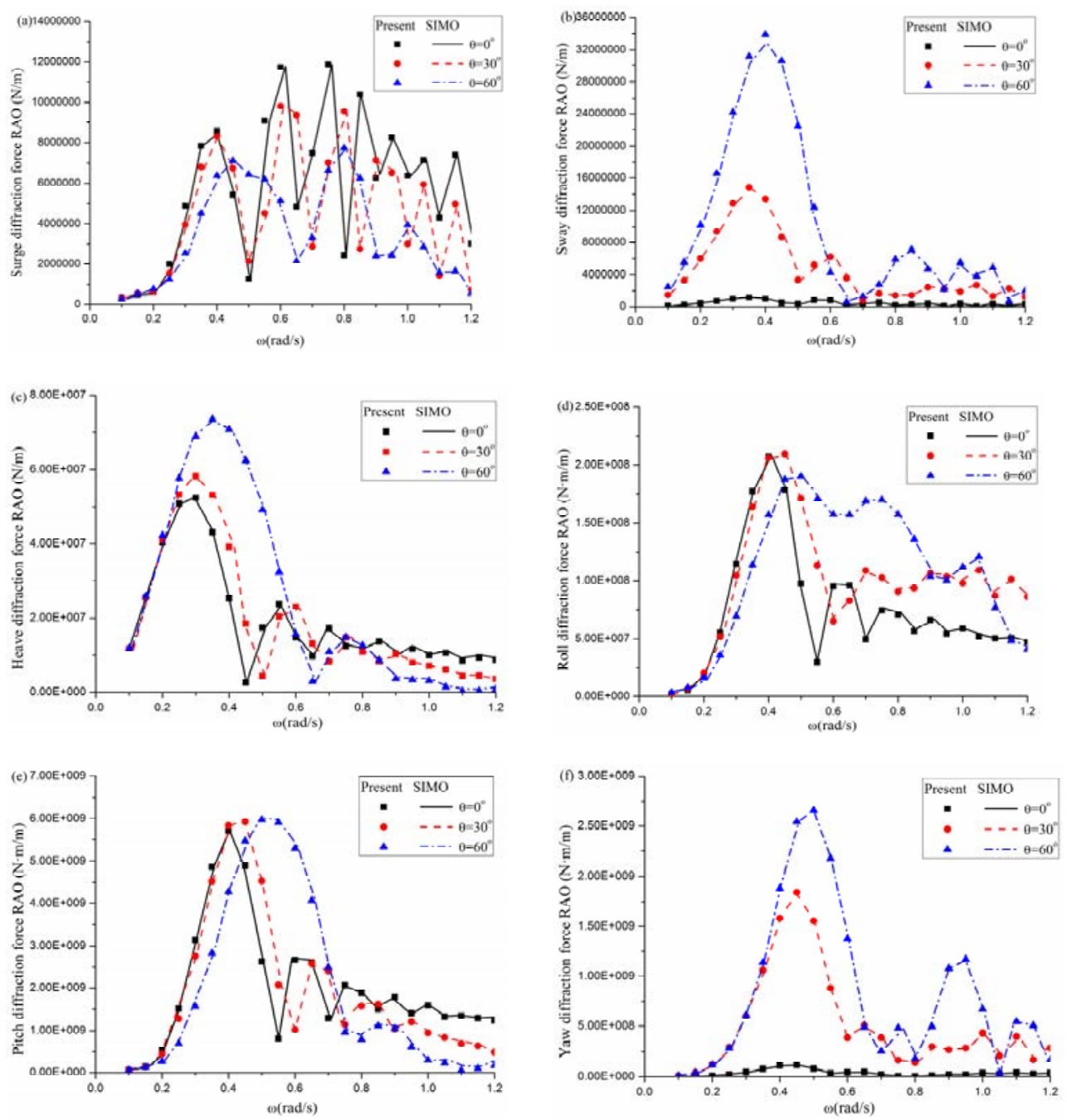

Fig. 8 The diffraction forces of the FPSO in the frequency domain as functions of wave frequency and incident wave angle. (a) surge (b) sway (c) heave (d) roll (c) pitch (d) yaw

\subsection{Effects of MWA system}

In order to investigate the effects of the MWA system on the motion responses, mooring/riser tensions of the FPSO, numerical simulations under the same environmental conditions are conducted without considering the existence of the MWA, for comparison. Figs. 9-10 show the response comparisons of the single body model and multi-body model in time domain as well as corresponding response spectrums in the frequency domain. The sway and yaw power spectral curves for fully coupled model are magnified to acquire better and clearer display effects. The maximum responses (MAX), standard deviations (STD) of low frequency (L-F) motion and wave frequency (W-F) motion are listed in Tab. 7. It is noted that frequency ranging from 0 to 0.3 and 0.3 to 1.6 denote the L-F and W-F motion, respectively. The figures and table suggest that the L-F motion which dominates the total horizontal responses (surge, sway and yaw motion), is completely controlled by damping and added mass induced from MWA, tension legs and risers for fully coupled model. Thus, the ratios of standard deviations of surge, sway and yaw L-F motion for single body model and multi-boy model are 2.25, 5.6 and 5.50, respectively, which also means the MWA system can obviously reduces the cumulative fatigue damage. As can be seen from Fig. 
9c, the heave equilibrium position of FPSO for multi-body model is smaller than the one of single body model, which is due to the reduction of the top tension of flexible risers, however, the heave amplitudes for two models are almost identical. In addition, the heave responses are perfectly dominated by the W-F motion. Similar to the heave motion, the roll and pitch responses are also controlled by W-F motion. The standard deviations of roll and pitch W-F motion for multi-body model reduce $41.34 \%$ and $28.89 \%$, respectively, as compared with the single body model. The statistical values also reveal that except for heave motions, the MWA can reduces significantly the L-F and W-F standard deviations of the FPSO. This is because the MWA induce the additional horizontal restoring stiffness for FPSO and reduce the initial tension of flexible risers. Accordingly, in the case of extreme operating condition, the MWA system can be placed at desirable a submerged depth by tension legs and apparently improves the motion performance of FPSO. In this regard, the FPSO with an additional MWA mooring system may more suitable for a practical engineering viewpoint.
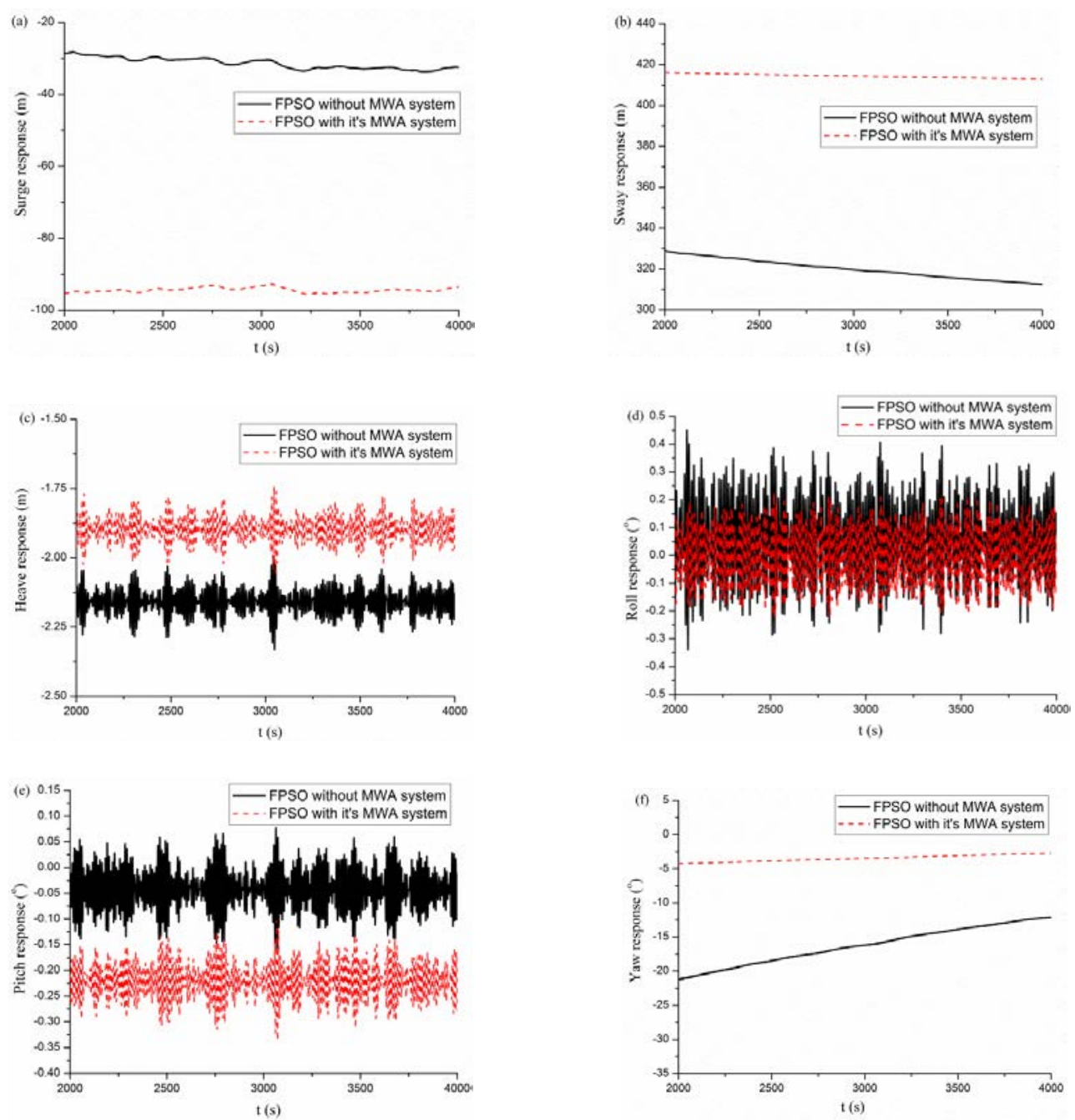

Fig. 9 Comparison of the motion responses of FPSO for two models. (a) surge (b) sway (c) heave (d) roll (c) pitch (d) yaw 

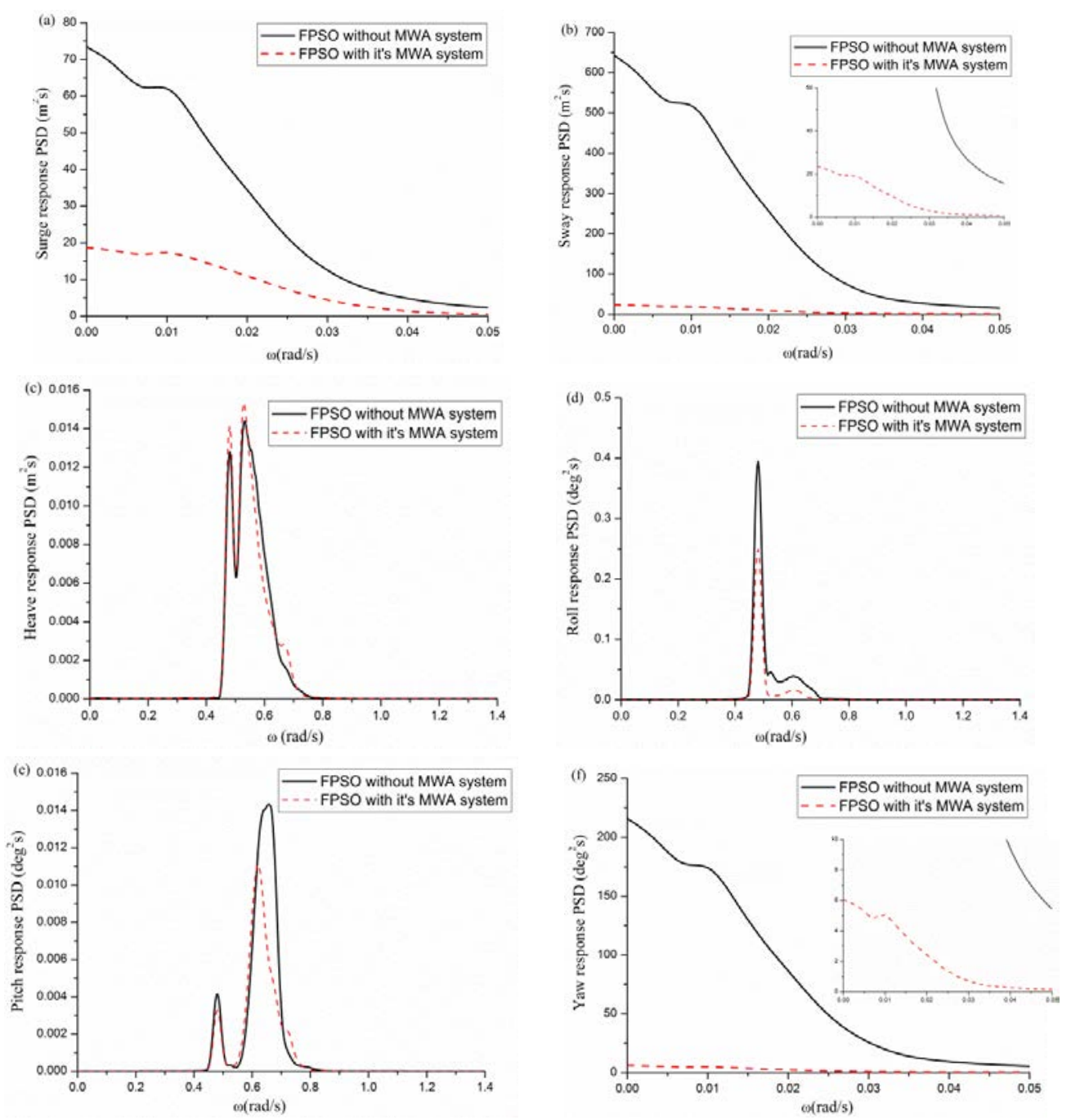

Fig. 10 Comparison of the response spectrums of FPSO for two models. (a) surge (b) sway (c) heave (d) roll (c) pitch (d) yaw

Tab. 7 Summary of motion statistics of the FPSO

\begin{tabular}{|c|c|c|c|}
\hline & & $\begin{array}{l}\text { FPSO with mooring } \\
\text { lines and risers system }\end{array}$ & $\begin{array}{l}\text { FPSO with its MWA, mooring } \\
\text { lines and risers system }\end{array}$ \\
\hline \multirow{3}{*}{ Surge (m) } & MAX & -83.411 & -95.711 \\
\hline & L-F STD & 1.304 & 0.584 \\
\hline & W-F STD & 0.148 & 0.032 \\
\hline \multirow{3}{*}{ Sway (m) } & MAX & 425.145 & 425.145 \\
\hline & L-F STD & 3.64 & 0.650 \\
\hline & W-F STD & 0.348 & 0.004 \\
\hline \multirow{3}{*}{ Heave (m) } & MAX & -2.315 & -2.111 \\
\hline & L-F STD & 0.001 & 0.001 \\
\hline & W-F STD & 0.004 & 0.004 \\
\hline \multirow{3}{*}{ Roll $\left(^{\circ}\right)$} & MAX & 0.563 & 0.212 \\
\hline & L-F STD & 0.032 & 0.002 \\
\hline & W-F STD & 0.179 & 0.105 \\
\hline
\end{tabular}




\begin{tabular}{rccc}
\hline \multirow{3}{*}{ Pitch $\left({ }^{\circ}\right)$} & MAX & 0.114 & -0.313 \\
& L-F STD & 0.004 & 0.002 \\
& W-F STD & 0.045 & 0.032 \\
\multirow{2}{*}{ Yaw $\left({ }^{\circ}\right)$} & MAX & -32.121 & -4.353 \\
& L-F STD & 1.921 & 0.349 \\
& W-F STD & 0.187 & 0.032 \\
\hline
\end{tabular}

Figs. 11-16 show the comparison of mooring line tensions calculated by the single-body model and multi-body model. Here, mooring lines $\# 1, \# 5$ and $\# 9$ are selected as the representative mooring of each group indicated in Fig. 2 in this analysis. In order to acquire better and clearer display effects, the W-F components are magnified in the tension spectrum figures. It can be found that the tensions of mooring line \#1 and \# 9 accounting the effects of MWA are smaller than that of single body model. Moreover, the maximum tensions of line \#1 and 9 for multi-body model decrease $39.56 \%$ and $41.78 \%$, respectively, as compared with the single body model according to data in Tab. 8. The MWA and flexible risers as well as the mooring lines \#5-\#8 in this paper are arranged at the negative $y$-direction, which potentially increases the mooring line \#5-\#8 tensions because the top pre-tension of risers can be resisted by the MWA system. Thus, the tensions of mooring line \# 5 for multi-body model are obviously larger than the results from single body model, and a special care has to be paid to the dynamic relationship between MWA, risers, and mooring lines in computing the multi-body hydrodynamic interaction. The total mooring line tension is simultaneously controlled by L-F and W-F tension, while the L-F tension is the major contributor for two models.

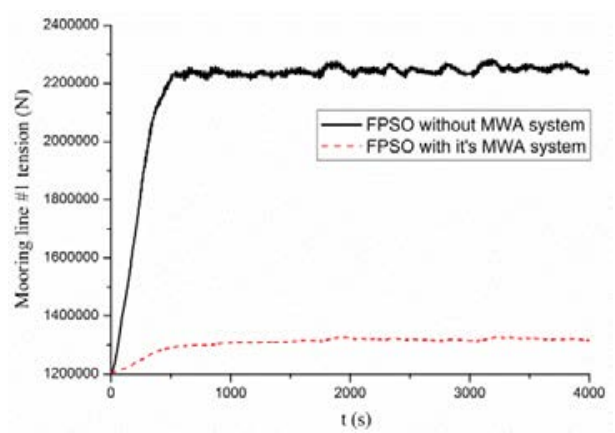

Fig. 11 The time series of mooring line \#1 tension

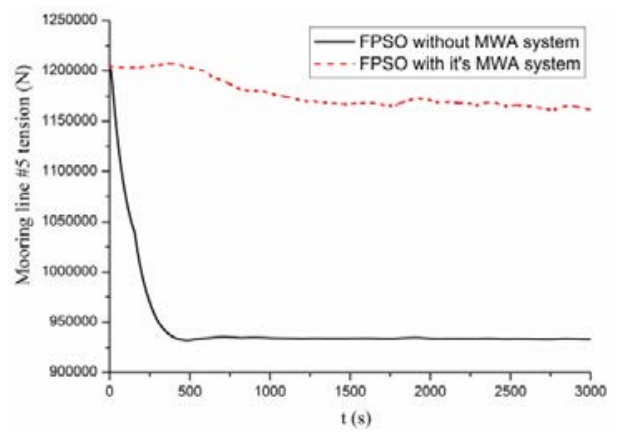

Fig. 13 The time series of mooring line $\# 5$ tension

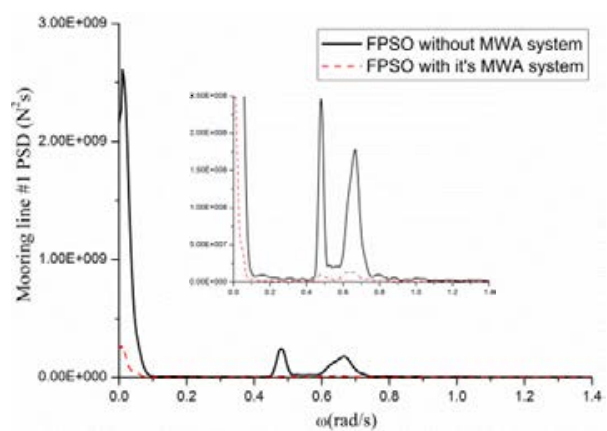

Fig. 12 The mooring line $\# 1$ tension spectrum

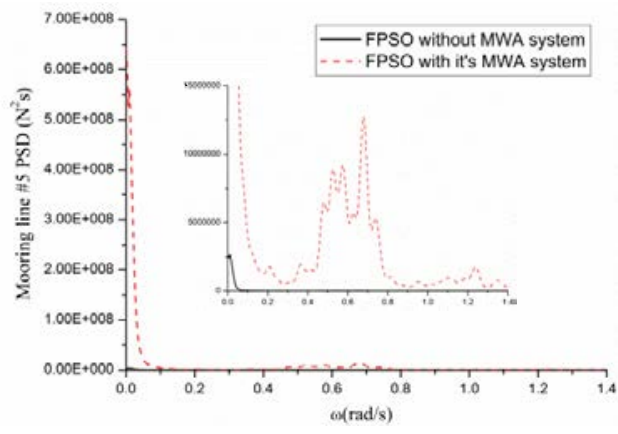

Fig. 14 The mooring line $\# 5$ tension spectrum 


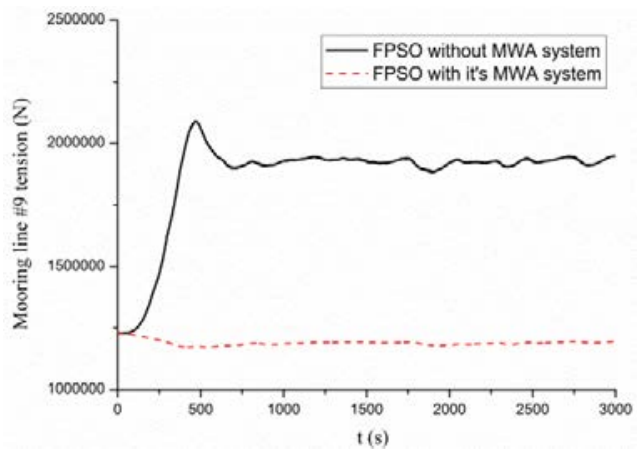

Fig. 15 The time series of mooring line \#9 tension

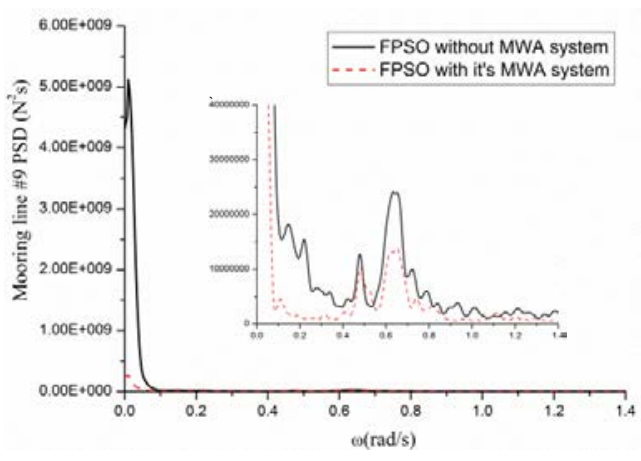

Fig. 16 The mooring line $\# 9$ tension spectrum

Tab. 8 Mooring tension statistics

\begin{tabular}{|c|c|c|c|c|c|c|c|c|c|}
\hline & \multicolumn{3}{|c|}{ Line \#1 $(\mathrm{N})$} & \multicolumn{3}{|c|}{ Line $\# 5(\mathrm{~N})$} & \multicolumn{3}{|c|}{ Line $\# 9(N)$} \\
\hline & MAX & L-F & W-F & MAX & L-F & W-F & MAX & L-F & W-F \\
\hline & & STD & STD & & STD & STD & & STD & STD \\
\hline Single & $2.25 \times$ & 9.76 & $5.65 \times$ & $1.21 \times$ & 2.87 & 45.6 & $2.13 \times$ & $1.27 \times$ & $2.73 \times$ \\
\hline FPSO & $10^{6}$ & $\times 10^{3}$ & $10^{3}$ & $10^{6}$ & $\times 10^{2}$ & 7 & $10^{6}$ & $10^{4}$ & $10^{3}$ \\
\hline FPSO with & $1.36 \times$ & 2.96 & $2.02 \times$ & $1.23 \times$ & 3.85 & 1.17 & $1.24 \times$ & $2.96 \times$ & $2.02 \times$ \\
\hline it's MWA & $10^{6}$ & $\times 10^{3}$ & $10^{3}$ & $10^{6}$ & $\times 10^{3}$ & $\times 10^{3}$ & $10^{6}$ & $10^{3}$ & $10^{3}$ \\
\hline
\end{tabular}

The MWA system also has influences on the top tension of flexible risers. Figs. 17-18 show the time series of the largest top tension among all flexible risers as well as the corresponding tension spectrum. It can be seen that the riser tension for multi-body model is clearly smaller than the results of single body model because the net buoyance induced by MWA resist top tension of risers and the tension legs provide some vertical restoring stiffness. Two important and valuable conclusions can be drawn through spectra analysis. First, the top tension of riser is controlled by both L-F and W-F tension, where the L-F tension is the major component for single body model and W-F tension dominates total tension when multi-body model is considered. Second, the both L-F and W-F STD for single body model are evidently larger than that of multi-body model. Thus, the dynamic behavior of flexible risers for single model sometimes can significantly influence the design and specially concern with the fatigue problem. These conclusions also can be obtained from Tab. 9, and the maximum tension value for multi-body model decreases $79.03 \%$ as compared with the single body model. It is evident that the MWA is very effective in reducing the dynamic responses of the flexible risers.

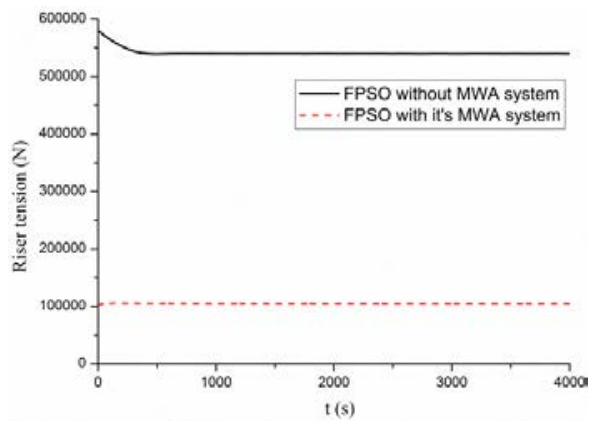

Fig. 17 The time series of riser tension

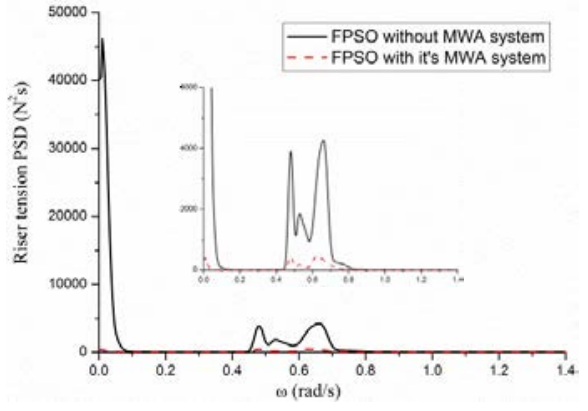

Fig. 18 The riser tension spectrum

Tab. 9 Riser tension statistics 


\begin{tabular}{cccc}
\hline & MAX & L-F & W-F \\
& & STD & STD \\
\hline Single model & $5.78 \times 10^{5}$ & 37.94 & 24.35 \\
FPSO with it's MWA & $1.17 \times 10^{5}$ & 3.16 & 8.03 \\
\hline
\end{tabular}

\subsection{Motions of MWA}
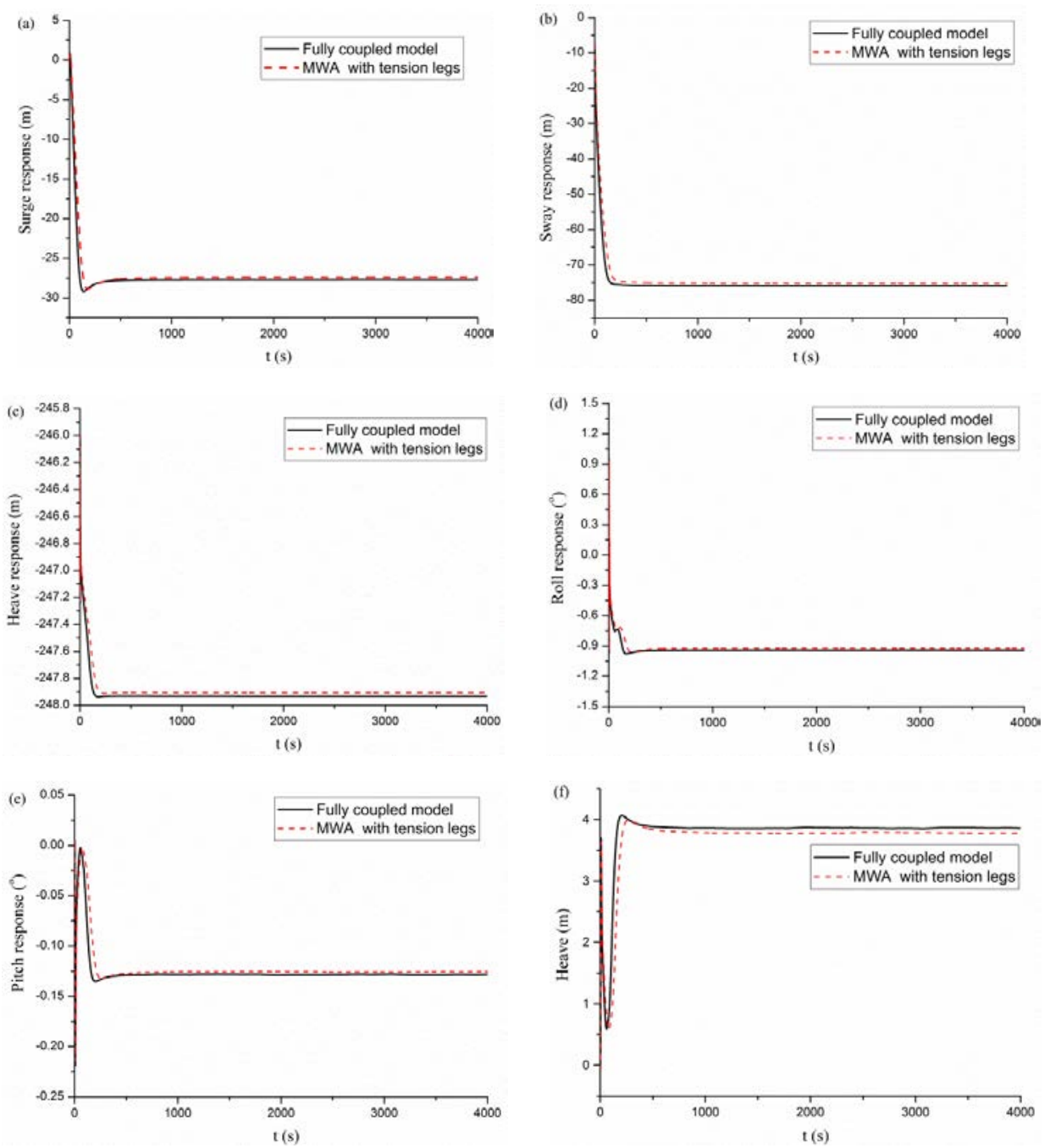

Fig. 19 The time series of MWA motion for fully coupled model and single MWA model with tension legs. (a) surge (b) sway (c) heave (d) roll (e) pitch (f) yaw

Fig. 19a-f shows the six degrees of freedom responses of MWA. In order to illustrate the effects of FPSO, mooring lines and tension legs on the motion responses of the MWA, the responses of single MWA model with tension legs also are plotted in these figures for comparison. Here, the weight of single MWA model needs to be modified to achieve identical top pre-tensions of tendons between two models. As can be seen from these figures, the motion response of MWA reaches its maximum value at around $t=400 \mathrm{~s}$ and then stay almost the same value with the increase of $t$. This is due to the dominant current and the additional restoring stiffness induced by tension legs. In addition, the calculated results from single MWA model with tension legs are slightly smaller than the results from fully coupled model. Thus, the dynamic response simulation of the single MWA model with tension legs having the same pre-tensions can be conducted to 
preliminary design and analysis of MWA structure. In addition, the results of both fully coupled model and single MWA model indicate that the motion responses of FPSO and mooring/riser tension are weakly dependent on the motion of MWA.

\section{Conclusion}

A fully coupled hull, mooring line, riser and tendon model is established and applied to the dynamic analysis of an FPSO with its MWA system under the combined parallel wave, wind and current action. The following conclusions are obtained from this study:

(1) Through the coupled analysis, the MWA system demonstrates the advantage of suppressing motions of FPSO (except heave). In addition, the horizontal motion i.e. surge, sway and yaw and vertical motion i.e. heave, roll and pitch are dominated by L-F and W-F motion, respectively.

(2) The top tensions of mooring lines which and MWA are at the different sides of FPSO are obviously smaller than that of single body model without considering the effects of MWA, however the tensions of mooring lines at the same side of the FPSO are magnified due to the tension reduction of risers. The L-F and W-F tensions simultaneously control the mooring line forces, while the L-F component is dominant.

(3)Due to the existence of the MWA and its tension legs system, the tensions of flexible risers, which are dominated by both L-F and W-F components, would apparently decrease. Furthermore,the STD values of multi-body model are smaller than that of single body model, and significantly reduce the possibility of fatigue issues of flexible risers.

(4) In order to avoid the action of wind and wave, the MWA is placed at desirable submergence depth and its motion responses aremaintainedat"steady state", which means that the simulation of single MWA model with tension leg mooring system can be performed topreliminary structural design and dynamic analysis of MWA.

In general, the numerical results obviously indicate the desirable motion performance and confirm the feasibility of the FPSO and its MWA system under classic working conditions. The present numerical model can be extended straightforwardly to the non-collinear environmental loads in a future study.

\section{Acknowledgment}

The authors are grateful to the National Science Foundation of china (Grant No. 51579122) for supporting this work.

\section{References}

[1] Kannah TR, Natarajan R. Experimental investigation of an external turret moored FPSO system by VALM arrangement. Ships and Offshore Structures 2006; 1(3):199-212.

[2] Wichers JEW,Develin PV. Effect of coupling of mooring lines and risers on the design values for a turret moored FPSO in deep water of the gulf of Mexico. In: Proceedings of 11th int. offshore and polar eng.Conference, ISOPE, 2001; 3:480-7.

[3] Lee DH. Nonlinear stability analysis and motion control of tandem moored tankers. Ph.D. Dissertation.Naval Architecture and Ocean Engineering, Seoul National University, Seoul, 2002.

[4] Fan TH, Qiao DS, Ou JP. Innovative approach to design truncated mooring system based on static and damping equivalent. Ships and Offshore Structures 2014; 9(6): 557-568. 
[5] Zhang L, Lu HN, Yang JM, Peng T, Xiao LF. Low-frequency drift forces and horizontal motions of a moored FPSO in bi-directional swell and wind-sea offshore West Africa. Ships and Offshore Structures 2013; 8(5): 425-440.

[6] Tahar A, Kim MH.Coupled-dynamic analysis of floating structures with polyester mooring lines. Ocean Engineering2008; 35: 1676-1685.

[7] Yang M, Teng B, Ning D, Shi Z. Coupled dynamic analysis for wave interaction with a truss spar and its mooring line/riser system in time domain. Ocean Engineering2012; 39:72-87.

[8] Korloo J, Black J, Wu C,Treu H. Design and Analysis of West Seno Floating Structures, OTC, Paper 16523, 2004.

[9] Fang MC,Kim CH.Hydrodynamically coupled motions of two ships advancing in oblique waves. Journal of Ship Research1986; 30(3):159-171.

[10] Huijsmans RHM.Diffraction and radiation of waves around side-by-Side moored Vessels. Proceedings of the Eleventh (2001) International Offshore and Polar Engineering Conference,406-412.

[11] Buchner B, Dijk A,Wikse J.Numerical multiple body simulations of side by side mooring to an FPSO. Proceedings of the 11th International Offshore and Polar Engineering Con- ference, Stavanger, Norway, 2001,pp. 343-353.

[12] Koo BJ, Kim MH. Hydrodynamic interactions and relative motions of two floating platforms with mooring lines in side-by-side offloading operation. Applied Ocean Research 2005; 27: 292-310.

[13] Zhao WH, YangJM, Hu ZQ.Hydrodynamic interaction between FLNG vessel and LNG carrier in side by side configuration. Journal of Hydrodynamics 2012; 24(5):648-657.

[14] Zhao WH, Yang JM, Hu ZQ, Wei YF. Full-scale measurement investigation of the hydrodynamic of a turret moored FPSO in a typhoon and deduction of its mooring loads. Ships and Offshore Structures 2012; 7(3):285-295.

[15] Zhao WH, Yang JM, Hu ZQ,Xie B. Hydrodynamic of an FLNG system in tandem offloading operation. Ocean Engineering 2013; 57:150-162.

[16] SIMO PROJECT TEAM. SIMO-theory manual Version 3.6, rev:2[M]. Trondheim, Norway: MARINTEK 2009. 